Cahiers de recherches médiévales

\title{
Le poison chez les Trastamare
}

De l'empoisonnement réel à l'imaginaire de l'empoisonnement

\section{Flora Ramires}

\section{(2) OpenEdition \\ Journals}

Édition électronique

URL : https://journals.openedition.org/crm/11504

DOI : $10.4000 / \mathrm{crm} .11504$

ISSN : 1955-2424

Éditeur

Honoré Champion

Édition imprimée

Date de publication : 15 juin 2009

Pagination : 53-69

ISSN : $1272-9752$

Référence électronique

Flora Ramires, "Le poison chez les Trastamare », Cahiers de recherches médiévales [En ligne], 17 | 2009, mis en ligne le 15 juin 2012, consulté le 15 décembre 2022. URL : http:// journals.openedition.org/crm/11504; DOI : https://doi.org/10.4000/crm.11504

Ce document a été généré automatiquement le 15 décembre 2022.

Tous droits réservés 


\title{
Le poison chez les Trastamare
}

De l'empoisonnement réel à l'imaginaire de l'empoisonnement

\author{
Flora Ramires
}

1 Le crime de poison en Castille n'a pas encore été suffisamment étudié. Seuls quelques rares chercheurs ${ }^{1}$ se sont aventurés dans ce domaine qui apparait comme bien problématique de par le manque de sources et le caractère parcellaire de ces dernières. Cependant, il nous semble nécessaire de tenter de comprendre les enjeux politiques et idéologiques de la pratique de la toxicatio dans cette aire géographique souvent oubliée

2 Nous nous proposons d'effectuer une typologie des crimes de poison à partir des références que nous avons pu relever dans des textes historiographiques de l'époque Trastamare, dynastie illégitime, arrivée à la suite d'un crime fratricide (l'assassinat de Pierre $\mathrm{I}^{\text {er }}$ dit le cruel par son demi-frère Henri II en 1369). Nous avons choisi de nous intéresser à cette époque car elle est marquée par des crimes politiques en tout genre. Elle débute par un fratricide qui élimine le roi et le remplace par un autre, dans une violence qui est dévoilée, assumée. Or, le crime de poison fait généralement partie du cercle privé et secret. Cette dynastie représente dans l'imaginaire collectif un point culminant de la violence politique, qui s'exprime par l'assassinat du rival, sans l'intervention du poison, ni de quelconque intermédiaire. Dans quelle mesure l'attitude du premier roi Trastamare Henri, se retrouve-t-elle dans la chronique de Pedro Lopez de Ayala? Ses successeurs feront-ils preuve de la même affirmation violente du pouvoir? Quelle tendance se dégage de ces chroniques?

3 Nous souhaiterions parvenir à évaluer les mécanismes et la réalité de ce phénomène dans le royaume de Castille à partir d'une approche diachronique et pluridisciplinaire. Il s'agira dans un premier temps de chercher à comprendre pourquoi les chroniqueurs choisissent de faire allusion à un empoisonnement et dans le cas d'une réponse positive, si l'identité de l'empoisonneur est mentionnée. Et alors, pour quelles raisons celui qui empoisonne le fait-il ? Les justifications politiques prédominent-elles? Enfin, le roi est-il le plus souvent victime ou auteur de ces crimes? 


\section{Une réalité événementielle : les rois en danger}

$4 \quad$ Nombreux sont les cas de morts mystérieuses ou inexplicables que l'on trouve évoquées dans les chroniques des $\mathrm{XIV}^{\mathrm{e}}$ et $\mathrm{XV}^{\mathrm{e}}$ siècles. Cette branche bâtarde de la branche castillane que l'on a coutume d'appeler Trastamare s'est caractérisée par un emploi de la violence qui a su lui garantir un avenir sur le trône de Castille. Cette dynastie plus qu'une autre est entourée de morts par poison qui entretiennent le mythe d'une famille aux coutumes toxiques que nous allons tenter de dévoiler.

5 Au commencement de la Chronique d'Henri II de Trastamare (1369-1379²), en 1370, soit un an après sa prise de pouvoir, Pedro Lopez de Ayala évoque la mort de Don Tello seigneur de Biscaye. Le titre qui précède cette notice indique un élément troublant: «Chapitre IV. Comment mourut Don Tello, seigneur de Biscaye, et comment l'Infant Jean, fils du Roi Henri, eut la Seigneurie. $»^{3}$ Cette note n'a rien d'anodine, le chroniqueur a conscience que cette mort est politique. Dès le titre nous savons qu'il s'agit d'une stratégie politique qui vise à asseoir le pouvoir royal d'un point de vue territorial. Ainsi, rien d'étonnant à ce que cette mort soit mise en relation avec un crime de poison. Le chroniqueur se fait l'écho des rumeurs : «Et certains disaient qu'on lui avait donné des herbes, et que celui qui les lui avait données était un médecin, que l'on disait maître Romain, qui était le médecin du roi Henri et qu'il les lui avait données par ordre du Roi. $»^{4}$ Il n'y a donc aucun doute sur le rôle du Roi dans cette affaire. Nous connaissons l'empoisonneur, il ne nous manque plus que les raisons de ce crime. Et notre chroniqueur de les décrire : «Parce que Don Tello était toujours en relation avec tout ceux qu'il savait ne pas souhaiter du bien au roi Henri ; mais tout cela n'est pas vrai, ce n'est que la rumeur qui dit que cela se passa ainsi. ${ }^{5}$ Notons que ce noble, qui est le frère du roi, est soupçonné de traîtrise envers son roi. Ce qui en soit justifierait la mort, mais alors, pourquoi utiliser le poison? Ne s'agirait-il pas d'un moyen réservé à une élite, aux plus nobles, ou la mort par poison rendant difficile la connaissance du meurtrier, n'éviterait-on pas ainsi des représailles? Face à cette rumeur, notre chroniqueur prend ses distances et finalement il en nie les fondements. Mais alors pourquoi avoir relayé cette information? Le doute reste, le roi ne serait-il pas un empoisonneur politique qui éliminerait ainsi ses opposants?

6 On ne retrouve pas d'autres évocations d'empoisonnements politiques. La seule référence au poison que l'on ait pu relever apparaît au moment de la mort du roi Henri, où le chroniqueur se fait l'écho, une nouvelle fois, d'une rumeur. Le roi aurait été empoisonné en 1379. Au sein même de la chronique nous pouvons lire que cette mort survint après douze jours d'agonie et de douleurs : « Le roi Henri, après que le roi de Navarre partit de Sancto Domingo, ne se sentit pas bien car il était malade, et subitement, il en fut très touché. $»^{6} \mathrm{La}$ référence à un possible empoisonnement apparaît mentionnée dans une note de Zurita qui développe ce pressentiment. La mort du roi serait due à un acte de traitrise : «Sur la rumeur selon laquelle le roi serait mort après s'être mis des brodequins empoisonnés que lui aurait présentés un adalid du Roi de Grenade Mohammed le Vieux, qui avait feint d'être un fugitif. $»^{7}$

7 L'empoisonnement est ici décrit comme un acte perfide perpétré par un ennemi, un étranger et un traître, à l'encontre du roi. Le poison passe par un objet. Notons que le procédé est identique dans le sortilège. Des correspondances dans le modus operandi existent entre la sphère de la magie et celle du poison. Le lien entre ces deux sphères peut se justifier de par l'origine de l'empoisonneur. En effet, venus généralement 
d'Orient, les maures ont la réputation d'être de redoutables sorciers ou magiciens. Il en va de même pour les juifs. Nous sommes bien évidemment en présence d'un témoignage sur la peur de l'empoisonnement chez les chrétiens qui se manifeste par la peur d'une conspiration contre la chrétienté. Cette conspiration imaginaire est à la source d'un discours de propagande que Pedro Lopez de Ayala emploie afin de renforcer le pouvoir en stigmatisant un ennemi qui n'est pas si imaginaire que cela...

Disons en outre que le chroniqueur classe cette information à un niveau de véracité inférieur à celui de l'exemple précédent. La rumeur n'est pas ici une source fiable pour le chroniqueur, mais à la différence de l'exemple antérieur, il ne l'intègre pas dans la structure du texte même. Toutefois, il s'agit d'un phénomène lié à l'imaginaire collectif des morts violentes. La violence de l'agonie du roi est ainsi interprétée comme un signe d'empoisonnement. Pour séduisant que soit ce signe, il ne fait partie que de la sphère publique des rumeurs pour le chroniqueur. Notons tout de même que cette note se termine par un renvoi à la mort du roi Henri III (1390-1406).

Il est fréquent de retrouver des allusions à l'empoisonnement au contact d'un objet empoisonné généralement offert par un Maure. La lettre d'un ancien Maure au roi contenue dans la Chronique de Jean II de Castille (1407-1454) d'Alvar García de Santa María, est éclairante sur ce point. En effet, il s'agit du témoignage d'un habitant de Priego, ancien Maure, qui avertit l'infant Ferdinand de Antequera - régent et oncle du jeune roi Jean II de Castille -des pratiques des rois maures. Écoutons-le :

Seigneur: Vous devez vous garder des présents et choses que vous amènent ces Maures, parce qu'ils pensent toujours à mal, comme ils sont pleins de mauvaises intentions et sans peur de Dieu, et qu'ils convoitent votre vie. Je me dois de vous avertir sur ce que j'ai pu voir et entendre à Grenade ; parce que Seigneur, je veux surtout vous avertir et agir selon ce que votre altesse voudra.

Seigneur, je vis par mes yeux à Grenade comment au roi Yuçaf - père de ce roi de Grenade qui maintenant règne -, fut envoyée par le roi de Fez une casaque ; et dès qu'il la vêtit, il se sentit aussitôt comme empoisonné, il mourut après trente jours, sa peaux tombant en morceaux. Et d'autre part Seigneur, sachez que ce roi Mohammed, frère de ce roi qui maintenant règne, mourut avec une chemise recouverte d'herbes qui lui fut donnée dans son bain.

Et de plus, Seigneur, lorsque j'étais à Grenade je vis que le roi Mohammed le Vieux envoya secrètement au roi Henri votre grand-père le gouverneur Pipes en disant qu'il venait d'être banni par son roi, dès que ce roi sut que le roi Henri votre grandpère voulait entrer en guerre avec lui l'année où il mourut. Et ce gouverneur Pipes lui amena beaucoup de présents, beaucoup de bijoux et de perles; parmi ces présents, il amena de très jolis brodequins qu'il paya bien cher car ils étaient empoisonnés. Peu de temps après les avoir portés, il mourut, en disant qu'il mourait de la goutte ; mais après on raconta dans tout Grenade qu'il avait été empoisonné par la plante des pieds. Et puis, Seigneur, le roi don Alonso qui mourut à Gibraltar, a la réputation dans tout Grenade d'avoir été empoisonné par les maures...8

Il s'agit donc d'une réelle croyance en une pratique politique. D'autres textes historiographiques enfin attestent de la croyance en ces Maures empoisonneurs. Nous avons retrouvé une référence à cette peur dans la Chronique des Rois Catholiques ${ }^{9}$, de Hernando del Pulgar (années 1480-1484) :

Et comme il y avait tant de gens qui arrivaient de toutes parts au camp, il y avait dans la troupe beaucoup de malades, et les gens étaient fatigués des souffrances qu'ils enduraient et des luttes qu'il y avait toujours contre les Maures. Et comme il y avait beaucoup de fagots qui étaient déjà secs, et ayant peur d'un quelconque feu qui par hasard s'allumerait, ou qui serait mis par les Maures mudéjares qui étaient dans le camp; et comme l'on craignait aussi un quelconque venin qui serait jeté 
dans les puits où les gens venaient boire de l'eau. Le roi et la reine, pour ces raisons, ordonnèrent que tous les Maures mudéjares sortissent du camp. ${ }^{10}$

11 Revenons au passage de la chronique de Jean II de Castille, où Alvar García de Santa María se fait l'écho de ces peurs, il met ainsi en garde les rois chrétiens d'avoir des relations avec les rois maures, tout en les mettant en garde contre les mauvais chrétiens :

Et par conséquent, tous les rois et grands seigneurs doivent se garder de telles choses, en particulier des gens mauvais sans foi ni loi qui cherchent toutes les façons possibles par lesquelles ils peuvent tuer avec des herbes ceux contre qui ils combattent. Et tous ceux qui désirent grandement conquérir les Maures, et leur faire la guerre, se doivent d'être prudents avec raison dans le manger et dans le vêtir, et que toutes ces choses soient goûtées. Car il n'est pas étrange de voir les Maures en user, car les mauvais chrétiens en usent aussi, quand ils veulent faire du mal à d'autres, pour atteindre leur honneur et statut, ils pensent tuer avec des herbes. ${ }^{11}$

L'empoisonnement n'est donc pas seulement une affaire liée à l'Autre, à ce nonchrétien, mais bien de façon plus globale à toute personne désireuse d'atteindre les " honneurs et le statut » de la personne empoisonnée. Ce qui revient à dire que toute personne ayant un statut élevé peut devenir potentiellement une victime. Le jugement de valeur qu'introduit l'expression "mauvais chrétien » est bien la marque d'une généralisation d'une pratique politique meurtrière.

L'empoisonnement supposé de ce roi est mis en relation avec une douleur à l'estomac. Le crime de poison est évoqué avec le même procédé, soit en s'appuyant sur la fama, la rumeur. À la différence de la mort d'Henri II, Henri III succombe suite à l'absorption d'un poison, et non par contamination au contact d'un objet empoisonné. Dans le deuxième cas, le chroniqueur s'attarde étrangement sur les événements qui précèdent cette mort. Écoutons-le :

Le roi étant à Valence, il tomba très gravement malade d'un point de côté, et le fils d'une de ses nourrices lui dit qu'il avait eu lui aussi cette maladie, et qu'il avait guéri grâce à des eaux de jusquiame extraites par distillation qu'il avait bues trois fois tous les trois jours, et que grâce à cela il avait guéri trois ou quatre malades de cette même maladie; et le roi voulut savoir si cela était vrai, ces derniers [médecins] lui répondirent que oui, et qu'il fallait que tous les neuf ou dix jours après avoir bu ce breuvage il restât au lit; et comme les médecins lui demandèrent et lui admonestèrent qu'il ne bût pas de cette eau, lui expliquant que c'était quelque chose de très fort, et que ceux qui avaient guéri grâce à elle étaient des hommes robustes et de meilleure constitution que lui, et qu'à cause de cela il ne devait en aucun cas la boire. Le roi voulut malgré tout tester sur lui-même cette expérience. Et après avoir bu cette eau il n'hésita pas à se lever. Une fois, allongé sur son lit, il était si souffrant qu'il resta sans pouls pendant plus d'une heure et dans toute la ville la rumeur se propagea qu'il était mort. Et afin qu'ils crussent le contraire on le mit à l'une des fenêtres de son Palais pour que tous pussent le voir. Après avoir bu cette eau le roi n'alla plus jamais bien jusqu'à ce qu'il mourût. Et certains disent que des herbes lui furent données, et d'autres que cela fut la cause de sa mort. ${ }^{12}$

Nous assistons à la description de la concoction d'un poison. Si tout médicament peutêtre un poison, celui-ci y ressemble à s'y méprendre. La jusquiame évoquée ici, peut avoir des propriétés calmantes et narcoleptiques tout en produisant une substance venimeuse qui attaque généralement le système nerveux. Tout dépend donc du dosage. Et notre chroniqueur évoque cette ambiguïté avec soin. Rappelons que cette plante apparaît dans certains traités de médecine de cette époque : ainsi elle peut parfois être utilisée comme composant dans certains médicaments. On retrouve effectivement 
certaines références à l'utilisation de cette plante dans certains traités de médecine ${ }^{13}$ contre les douleurs intestinales entre autres ${ }^{14}$. Nous reviendrons sur ce point dans notre deuxième partie.

Parvenue à ce point, qu'il nous soit permis de tenter un grand écart chronologique ; à l'instar d'Henri III, le roi Philippe le Beau fut probablement empoisonné. En septembre 1504 Philippe le Beau se sentit indisposé après une partie de chasse, il commença à avoir de la fièvre, des frissons et une éruption cutanée. Bien qu'apparemment tous les médicaments et aliments qui étaient administrés au patient fussent goûtés par sa femme Jeanne, il mourut. Les soupçons se tournèrent alors vers sa femme et son beaupère Ferdinand le Catholique. Ce dernier mourut en 1516, dans des circonstances plutôt étranges. L'un des chroniqueurs ${ }^{15}$ des Rois Catholiques fait une allusion rapide à une rumeur d'empoisonnement concernant le roi Ferdinand II d'Aragon après son remariage avec Germaine de Foix. Il indique qu'il serait tombé malade après avoir bu un breuvage de fertilité que lui aurait donné la reine :

Et la cour étant dans cette ville, durant le mois de mars, et le roi Ferdinand à Carrioncillo, lieu éloigné de Medina d'une lieue, lieu agréable et abondant en gibier, il se reposait avec la reine Germaine sa femme; en conséquence son Altesse eut tellement envie d'avoir une descendance, en particulier un fils qui hériterait des royaumes d'Aragon que la reine lui fit donner certains breuvages à base de testicules de taureau et de choses de médecine qui aidaient à avoir une descendance ; car on lui laissa entendre que leur action serait immédiate. Même si certains pensèrent que du poison lui avait été donné ${ }^{16}$.

16 Ainsi, Germaine de Foix voulant améliorer les performances de son époux, demanda à Isabel de Velasco de préparer un breuvage à base de testicules de taureau, et de cantharidine. Rappelons que la cantharidine est une substance tirée d'un insecte, la mouche dite d'Espagne ou de Milan, célèbre depuis l'Antiquité pour ses propriétés aphrodisiaques: une poudre faite à partir de l'insecte était reconnue alors comme propice aux érections. Cependant, une surdose peut être mortelle. Ferdinand n'en réchappa pas. Surdose involontaire ou empoisonnement, le doute plane dans les écrits historiographiques d'alors.

\section{Les traités de médecine et autres textes se référant au poison : la connaissance sur les poisons entre superstitions et croyances ; état de la question au $\mathrm{XV}^{\mathrm{e}}$ en Espagne}

17 Ne pouvant pas nous livrer, par la force des choses, à une analyse en profondeur de ces traités, nous avons décidé de nous intéresser aux textes contenus dans la base de données Corde $^{17}$ afin d'esquisser une typologie des poisons auxquels on se réfère en cette fin de moyen âge dans les traités de médecines et autres documents scientifiques d'alors en Castille. On relèvera ici une réelle préoccupation autour du poison et de la connaissance de la science du poison. On voit apparaître de nombreux traités dans lesquels on décrit une typologie des poisons. Rappelons qu'il existe trois types de poison, les poisons d'origine végétale, les poisons d'origine minérale et enfin les poisons d'origine animale. Ces différentes catégories se retrouvent dans les traités de médecine de cette époque. La réalité à laquelle elles renvoient est bien évidemment connue de tous. 
18 Nous avons été confrontée à la présence de la réalité médicale du poison. Un certain nombre de traités évoquent les symptômes dus à un empoisonnement. Ainsi, Francisco López de Villalobos, dans son Sumario de la medicina con un compendio sobre las pestíferas bubas $^{18}$, (1498), où il décrit différents types de poison et leurs remèdes :

$\mathrm{Au}$ sujet des venins et des poisons: N'importe quel poison contraire ou malin ou bien fait ce mal avec ses propriétés comme l'arsenic et le lièvre de mer ou bien sans ses propriétés il chemine vers la mort comme c'est le cas avec le napel. Avec ses propriétés, celui qui en prend ou en boit, sentira un goût et une horrible douleur et des nausées, et il perdra sa couleur. S'il s'agit d'un poison froid il sera tout endormi avec une grande froideur, paresseux et immobile. Recherchez les symptômes et soignez-le. Si le poison est chaud il aura une très grande soif, une très grande douleur, une très grande fièvre, de fortes brûlures et de la rage. S'il ne sent pas de froid ni de chaleur mais qu'il se sent affaibli et en sueur c'est par ses propriétés qu'il le tourmente; avec de l'eau chaude et une huile quelconque faites vomir l'empoisonné. Si le ventre au dessous de la douleur s'altère, mettez-y une aide en quelque manière; ensuite, boire du mithridate est très bon. Continuez le traitement, et si l'on sent le poison brûler, donnez à boire beaucoup de lait et d'eau froide, et recouvrez le ventre avec du santal et du camphre. Faites-lui manger des morceaux de roses. Si le venin est froid, donnez-lui de l'ail à manger et du vin. S'il a causé ce mal par ses propriétés, la thériaque pour cela est très adaptée et agit continûment sur lui avec ses agents cordiaux. Du poison du chien enragé ou de quelques autres animaux en cet état: si un animal ou un chien enragé empoisonne et corrompt, soignez-le ainsi. Laissez sortir beaucoup de sang de la plaie, si la plaie est petite qu'elle devienne plus grande, et abandonnez saignées et purges. Le mithridate et la thériaque en breuvage vous lui donnerez. Vous compresserez la plaie une fois qu'elle sera bien sèche avec de l'ail et des oignons, et dès que le venin est à l'intérieur, faites-le sortir par des purges, et continuez comme avec la précédente. Et pendant quarante jours, ne suturez pas la plaie, mais garrottez fortement entre la plaie et le cœur et pendant une année entière donnez-lui sa ration de quelque antidote ou d'une thériaque puissante. Et d'autres poisons je ne veux pas parler car pour ceux qui savent cela leur suffit, et si un méchant et un individu pervers avec des armes comme celles-ci voulait tuer, je ne veux pas les lui donner ici ${ }^{19}$.

19 On retrouve ici une réelle préoccupation quant à l'utilisation de connaissances liées au poison à des fins criminelles. L'autocensure est de rigueur, la méfiance à l'égard de l'utilisateur de ces connaissances est grande, d'où le refus de décrire certains poisons.

Notons que le poison est souvent associé à la peur. Ce qui a donné lieu à une littérature autour des méthodes de protection contre l'empoisonneur. À titre d'exemple nous renvoyons à un passage de l'Arte Cisoria, d'Enrique de Villena, daté de $1423^{20}$. Il y est fait référence à la croyance au pouvoir des pierres sur le poison: "Ses mains garnies de bagues avec des pierres ou des émaux efficaces contre le poison et l'air infect, tels que le rubis, le diamant, la jacinthe, l'émeraude, le corail, la licorne, la serpentine, le bézoard et la pyrope, celle que l'on fait à partir du cœur d'un homme mort avec du venin froid et cuit, si l'on souhaite endurcir ou lapidifier dans un feu réverbérant. Alexandre portait celle-ci entre toutes, selon ce qu'Aristote raconte dans son Lapidaire. $»^{21}$ Ce passage s'intègre à une thématique de l'éducation des princes (telles que l'hygiène des mains). On retrouve une autre référence au poison dans un passage concernant les couteaux et leurs étuis : «Et que le fourreau est une couverture comme un écritoire avec des cordons joliment travaillés. Et l'on peut les faire en cuir ; et s'il s'agit d'un cuir de cerf, c'est mieux, car il est contre le venin, et c'est pour cela que l'on en fait les liens en cas de morsures venimeuses. ${ }^{22}$ Le poison fait partie de l'imaginaire 
du pouvoir et sa présence dans des activités qui sont à n'en pas douter réservées à l'élite démontre, s'il était encore nécessaire, que le poison rôde autour des puissants. Il faut donc s'en préserver, d'où l'émergence de ces traités et autres textes visant à protéger les puissants dès leur plus jeune âge.

Malgré les efforts donnés, le poison est partout. Dans toutes les cours péninsulaires on relève des cas d'empoisonnement plus ou moins pris au sérieux.

\section{D'ouest en est : entourage en danger}

En poursuivant notre parcours des cas d'empoisonnement, nous allons aborder maintenant le cas du royaume de Navarre où une figure est souvent associée au poison. Il s'agit du Prince de Viana (1421-1461), fils né du premier mariage de Jean II avec Blanche de Navarre (1386-1441). Lorsqu'elle mourut en 1441, celle-ci laissa son royaume à son fils aîné, le jeune prince de Viana, mais elle le supplia de ne pas prendre ce titre du vivant de son père Jean II de Navarre. On a pensé à un possible empoisonnement de la reine comme cause du décès. D'ailleurs, les années qui suivirent furent marquées par des guerres civiles qui opposèrent le jeune prince à son père Jean II. Il mourut à l'âge de quarante ans d'une pleurésie, mais on raconte qu'il fut empoisonné par sa marâtre doña Juana Enriquez, fille de l'amiral de Castille. Sa sœur Blanche aurait subi le même sort, elle aurait été empoisonnée par sa sœur cadette Eléonore. Certains comme Loys de Mayerne Turquets ${ }^{23}$, firent retomber les soupçons sur un alchimiste juif qui vivait dans l'une des maisons du baron d'Aranprunyà. On parla même du prix du breuvage mortel d'une valeur de « 3.000 onzas de oro " prix qu'aurait satisfait Juana Enriquez. Certains historiens tels qu'Antonio Bofarull ${ }^{24}$, dans son Histoire critique sur la Catalogne affirment que l'identité du médecin était Juan Vezach, qu'il met en relation avec le comte de Pallars, capitaine général de l'armée catalane. Rappelons que les dernières années de la vie du Prince de Viana furent marquées par un délire paranoïaque de persécution qui lui faisait croire qu'on voulait l'empoisonner.

Avant de relancer notre progression, nous aimerions reprendre à titre d'exemple de cette obsession pour le poison, l'anecdote que Pelta reprend dans son ouvrage sur l'histoire du poison ${ }^{25}$. Il y fait allusion à une pratique courante dans les cours péninsulaires. Il raconte que le futur roi Ferdinand le Catholique dut goûter le repas de Charles de Viana, son demi-frère, lorsque ce dernier refusa de manger :

On raconte à ce sujet qu'une fois son demi-frère, le futur roi Ferdinand Le Catholique, dut 'goûter les mets [hacer la salva]' quand Charles de Viana refusa de manger. Le verbe 'salvar' fait référence à la coutume de goûter la nourriture et la boisson par une personne chargée de faire le service aux rois et aux grands seigneurs, afin d'écarter l'existence de venin dans ces dernières. ${ }^{26}$

Cette pratique révèle une conscience de la réalité du poison, tout en mettant en avant la peur que l'empoisonneur soit un proche. L'empoisonneur peut faire partie de l'entourage des puissants, il convient donc d'être vigilant d'où l'attitude du Prince de Viana.

Il est frappant de voir que les morts qui entourent l'arrivée au pouvoir sont généralement associées au poison. Elles sont des manifestations des conflits politiques et des rivalités qui règnent à l'intérieur même de la cour.

Quittons la Navarre et intéressons-nous maintenant à un récit privé à tendance historiographique. Nous avons pu constater ${ }^{27}$ qu'il y avait dans l'œuvre de Lope García 
de Salazar - souvent considéré comme le premier historien de Biscaye - Istoria de las bienandanças e fortunas ${ }^{28}$, un réel intérêt pour les crimes de poison. Là encore, nous avons relevé plus d'une cinquantaine de références au terme "yerbas» (herbes, poisons), dont quatre-vingts pour cent en relation avec des crimes politiques tels que la mort d'Eléonore de Guzmán. On ne sera pas étonnée que certaines morts liées au poison aient pour origine un ordre royal. De telle sorte que le poison est ainsi mis en relation avec une pratique que nous retrouvons à la genèse même des Trastamare.

À ce point de notre démonstration, il convient de rappeler que l'empoisonnement de la mère d'Henri II, Éléonore de Guzmán, tout comme celui de la jeune épouse du roi Pierre $\mathrm{I}^{\mathrm{er}}$, Blanche, permet de distinguer dans les pratiques politiques une tendance générique. Nous sommes tentée d'y voir une forme d'attitude politique vis-à-vis des femmes de pouvoir. Nous en voulons pour preuve le cas d'Éléonore de Guzmán :

L'histoire a raconté comment mourut le roi Alphonse dans le camp de Gibraltar et comment les castillans sortirent avec son corps et comment par la suite commencèrent les agitations. Dans la première année de son règne Éléonore de Guzmán, concubine du roi Alphonse et mère de ses fils, Henri, Frédéric, Tello et Sanche, fut arrêtée à Séville par la reine Marie, sur le conseil de Juan Alonso d'Alburquerque, majordome du roi, qui était parent avec la reine. Elle fut conduite au château de Talavera et empoisonnée. Et dès qu'elle fut emprisonnée, ses fils et ses proches, les Guzmán, quittèrent la cour, il s'en suivit beaucoup de mal en Castille sous le règne du roi Pierre, selon ce qui est raconté plus loin. ${ }^{29}$

On notera que ce même procédé d'élimination réapparaît dans la description de la mort de la reine Blanche :

Titre portant sur comment le roi Pierre fit assassiner la reine d'Aragon et Jeanne, [épouse du] seigneur de Biscaye et de Lara. La neuvième année du règne de ce roi Pierre, soit en 1369, il fit assassiner la reine d'Aragon à Castroxeriz, elle y était enfermée par dépit, son fils l'infant lui faisait la guerre, et il fit empoisonner Jeanne de Lara, femme du comte Tello, Seigneur de Biscaye, et Isabelle, sa sœur, femme de l'infant Jean, dans le château d'Almodovar del Rio, où elles étaient emprisonnées. Et avec elles, mourut la génération des seigneurs de Biscaye et de Lara. Et il fit également tuer avec des herbes la reine Blanche de Bourbon, sa femme, qui était dans le château de Medina Sidonia. ${ }^{30}$

Continuons notre parcours dans l'univers des rumeurs et des peurs d'empoisonnement. Nous observons que le poison fait partie des pratiques politiques puisqu'on le retrouve dans chacune des chroniques des rois Trastamare. Pas une n'échappe à ce constat. Ainsi, sous le règne de Jean $\mathrm{I}^{\mathrm{er}}$, peu avant la montée sur le trône d'Henri III, Éléonore la tante du futur Henri III, fuit la Navarre car elle accuse son mari Charles III de tenter de l'empoisonner avec l'aide d'un médecin juif ${ }^{31}$. Dans le même ordre d'idée, nous retrouvons au milieu du $\mathrm{XV}^{\mathrm{e}}$ siècle des rumeurs de morts par empoisonnement, entre autres avec le mystère qui entoure le décès de la reine Marie de Castille et celui de sa sœur Éléonore, reine de Portugal. À la différence de la chronique royale de Jean II de Castille, les Annales d'Aragon de Jerónimo de Zurita - historien aragonais du XVI ${ }^{\mathrm{e}}$ siècle évoquent ces morts et transmettent les différentes rumeurs qui les entourent sans détours. Un personnage pourtant réapparaît à plusieurs reprises, il semble être l'instigateur de l'empoisonnement, il s'agit d'Álvaro de Luna :

Morts des reines de Portugal et de Castille, sœurs du roi [d'Aragon]; les soupçons qu'elles causèrent et avec quels indices. La reine Éléonore de Portugal était décédée le 18 février de cette année alors qu'elle était dans la ville de Tolède dans le monastère de Santo Domingo el Real; il arriva au roi de Castille la nouvelle de sa mort lorsqu'il était à El Espinar. Et peu de jours après que le roi de Navarre passa les frontières, la reine de Castille mourut à Villacastín, petit village de la province de 
Ségovie. Et les gens étaient convaincus que des herbes lui avaient été données; et de cela il y eut beaucoup de signes, et on accusa le connétable Álvaro de Luna de s'être procuré avec son savoir le poison avec lequel moururent les reines et tous comprirent cela de par la mort fulgurante de la reine de Portugal, de par la célérité $\mathrm{du}$ poison avec lequel elle mourut, et de par tous les indices et signes d'empoisonnement qui se virent sur son corps après sa mort, et c'est avec le même poison que la reine de Castille, sa sœur, fut assassinée. Et cette rumeur fut causée par la conduite de ses princesses, qui en matière de vie honnête n'étaient pas de celles dont on pouvait dire le plus grand bien, et par la récente confédération qu'il y eut entre la reine de Castille et son fils pour sortir le roi, son époux, de l'influence du connétable. ${ }^{32}$

29 de poison. Nous y percevons en filigrane une arme politique privilégiée dans les crimes
contre les femmes de pouvoir:

Peine du roi [d'Aragon] pour la mort des reines ses sœurs. Lorsque le roi sut ce qui se disait au sujet de la mort des reines ses sœurs, il en eut une grande peine et il envoya dire à ses ambassadeurs que si jamais il s'avérait que la rumeur qui circulait partout était vraie, que la mort des reines de Castille et de Portugal avait été obtenue par ruse et méchanceté, qu'ils ne traitassent pas des choses contenues dans les instructions qu'ils portaient. Cela se déroula alors que le roi était à Naples le 27 mai, huit jours après qu'avaient été mises toutes choses en ordre de bataille, avant l'arrivée des ambassadeurs. ${ }^{33}$

Nul doute que cette pratique soit liée au secret réservé aux affaires qui mettent en jeu l'entourage royal, et finalement sans preuves d'empoisonnement la vengeance semble bien difficile!

En ce sens, les femmes sont certes des victimes du poison, mais ne tombons pas dans une visée trop généralisatrice, les hommes de pouvoir en sont également les victimes. Mais dans ce cas, la justification du crime passe alors par la notion d'ordre. Dès lors, l'assassinat politique est lié à une préservation de l'ordre publique. Et ceux qui ne respectent pas ce principe sont éliminés comme le sera Pedro Manrique :

Titre portant sur la prison et mort du gouverneur (adelantado) Pero Manrique. Cette année-là le roi Jean [de Castille] sut comment le gouverneur Pero Manrique pactisait avec Alphonse de Gijón et d'autres pour provoquer des troubles dans le royaume. Et pour cela et comme c'était un agitateur et un homme dépensant outre mesure, et qu'il gaspillait son bien et qu'il n'avait pas d'enfants, le roi le fit arrêter. Et lorsqu'il fut emprisonné au château de Plasencia, il mourut au bout d'un an; et on dit que ce fut par empoisonnement et que ce furent ses frères Gómez Manrique et Diego Gómez de Manrique qui organisèrent sa mort, pour cela le roi leur donna tous ses biens. ${ }^{34}$

31 Sous ce jour, l'empoisonneur apparaît avec une plus grande netteté. Nous constatons que dans le cas de châtiments royaux, il s'agit d'abord d'une décision juridique. Ensuite, le poison intervient comme un moyen efficace de faire taire le trublion. Prenons notre exemple, la référence aux frères de Pedro Manrique comme complices du crime, permet d'illustrer la part active jouée par l'entourage des puissants dans la pratique de l'empoisonnement. D'ailleurs, ne seraient-ils pas les auteurs de l'empoisonnement?

Revenons au récit de García Salazar, ce dernier évoque le cas du duc d'Arjona qui fut empoisonné sous Jean II de Castille :

À cette date en Castille, les rois Alphonse d'Aragon, Jean de Navarre, l'infant Henri, le comte de Castro et d'autres chevaliers de Castille qui étaient de leur parti, entrèrent en Castille ; le connétable et les autres chevaliers du royaume sortirent et se rassemblèrent à Monteagudo pour combattre ; et étant là-bas, ils signèrent la 
paix. Et chacun retourna sur ses terres, le roi Jean [de Castille] estimant qu'il avait été injurié par l'entrée des rois d'Aragon et de Navarre dans son royaume, rassembla ses hommes et ses chevaliers nobles, qui étaient fort nombreux pour entrer en Aragon et il monta le camp à Veramaçan. Et là on captura le duc d'Arjona, qui venait de Galice avec milles chevaux et beaucoup de fantassins, disant qu'il avait été mis au courant de l'entrée des rois d'Aragon et de Navarre, il fut emprisonné à Peñafiel où il mourut empoisonné. Le dit roi Jean entra en Aragon où il y fit beaucoup de mal et à son retour en Castille il répartit toutes les terres que les infants et le duc possédaient en Castille entre les chevaliers du royaume. Et la guerre continuait. ${ }^{35}$

Outre la présentation d'un vassal infidèle à son roi, c'est aussi à un roi en colère que nous avons affaire. L'ire du roi est sans appel, le poison en est la manifestation exemplaire. Dans ce contexte de guerre, et donc de tensions politiques, le poison n'apparaît pas moins représenté :

Titre sur comment le roi Jean fut arrêté à Médina del Campo et comment le connétable fuit à Escalona. À ce moment, le gouverneur Pedro Manrique fut arrêté et il s'enfuit de Fuerte Dueña; et en se rassemblant avec les chevaliers, ils firent exiler le connétable de la cour et celui-ci resta à Escalona jusqu'à ce qu'eux-mêmes le fissent revenir près du roi. Et lorsqu'il revient, des herbes furent données au gouverneur alors qu'il mangeait avec l'archevêque de Tolède, on prétendit que se fut par ordre du Maitre, son frère, et qu'il en mourut avec de grandes douleurs. ${ }^{36}$

Nous serions tentée de souscrire à un paradoxe, qui serait le suivant. Le poison est l'arme des puissants et des vainqueurs, mais constituerait-il une preuve de faiblesse ou d'intelligence politique? Ainsi, trouve-t-on des cas d'empoisonnements intercalés dans les récits historiographiques, réapparaissant dans les moments de crises du pouvoir comme une solution rapide et efficace aux troubles politiques. Dès lors, au-delà de l'imaginaire qu'il véhicule, le poison chez les Trastamare est à associer à une pratique politique polymorphe.

\section{Conclusion}

Dans notre position de départ, il s'agissait avant tout, rappelons-le, d'envisager les cas d'empoisonnement en relation avec une dynastie : les Trastamare, ce qui requérait une vision globalisatrice de cette période. Sans vouloir prétendre à l'exhaustivité nous avons tenté de dépeindre les cas d'empoisonnements politiques en relation avec la prégnance sur les esprits de cet imaginaire.

Sans pouvoir l'étayer suffisamment nous avons le sentiment qu'un nouvel état d'esprit est en train de poindre opposant le lien du sang au lien social et politique qui s'affirme sous cette dynastie. Le poison renvoie à des valeurs et des enjeux qui ont cours dans ces sociétés.

À y regarder de plus près, ce parcours nous a démontré qu'entre les embûches et la (sur)vie de cour, les femmes et les hommes de pouvoir se savent en danger, d'où la lucidité des témoins qui en évoquent les fins violentes.

Réalité, rêve, illusion ou simple manipulation propagandistique, le poison en Castille a encore bien des choses à nous révéler... 


\section{NOTES}

1. José Manuel Nieto Soria, Un crimen en la corte. Caída y ascenso de Gutierre Alvarez de Toledo, Señor de Alba (1376-1446), Madrid, Silex, 2006 ; Emilio Mitre, Una muerte para un rey, Enrique III de Castilla, Valladolid, Ambito, 2001 et du même auteur «De la toma de Algeciras a la campaña de Antequera », Hispania, XXXII, (1972), note 32, p. 86-87.

2. Nous faisons ici référence aux dates de début et de fin de règne.

3. «Cap. VI. Como morió Don Tello, Señor de Vizcaya, é como el Infante Don Juan, fijo del Rey Don Enrique, ovo el Señorio ", Cronicas de los Reyes de Castilla, Biblioteca de Autores Españoles, vol. 2, Madrid, éd. Cayetano Rosell, 1877, p. 7.

4. «é algunos decian que le fueran dadas hiervas, é que se las diera un Físico, que decían Maestre Romano, que era Físico del Rey Don Enrique é que se las diera por mandado de dicho Rey », loc. cit.

5. «Por razon que Don Tello andaba siempre tratando con todos aquellos que él sabía que non querian bien al Rey Don Enrique ; pero esto non era cierto, salvo la fama que fue así. », loc. cit.

6. «El Rey Don Enrique, despues que el Rey de Navarra partió de Sancto Domingo, non se sintió bien ca ovo una dolencia, é súbito fue muy afincado della. ", Crónicas de los Reyes de Castilla, vol. 2, p. 37.

7. «Sobre la fama de que el Rey Don Enrique murió de resultas de haberse puesto unos brodeguies envenenados que con varias joyas le presentó un Ad Lid del Rey de Granada Mahomad el viejo, fingiéndose fugitivo[...] ", Crónicas de los Reyes de Castilla, vol. 2, p. 61.

8. Alvar García de Santa María, Crónica de Juan II de Castilla, éd. Carriazo, Juan de Mata, Madrid, Real Academia de la Historia, 1982, 1409, p. 269-270.

9. Pulgar, Hernando de, Crónica de los Reyes Católicos, Juan de Mata Carriazo, Espasa-Calpe Madrid, 1943, p. 310.

10. «Y como concurrían gentes de tantas partes al real, avía en la hueste muchos enfermos, y la gente estaua fatigada de los trabajos que pasauan \& peleas que continuo avían con los moros. Y porque estauan fechas muchas ramadas, las quales estauan ya secas, reçelando de algún fuego que por caso se ençendiese, o que fuese echado por los moros mudéjares que andauan en el real ; \& asimismo se temía de algund veneno que se echase en los pozos del agua donde las gentes beuían. E por esta causa el Rey e la Reyna mandaron que todos los moros mudéjares saliesen luego del real. » (p. 310).

11. «E por ende, todos los reyes y grandes señores se deven mucho guardar de las semejantes cosas, especialmente de los malos sin ley que a los que les guerrean buscan quantas maneras pueden por los matar con yervas. $\mathrm{E}$ todos los que han gran voluntad de conquerir a los moros, e de les fazer guerra, es gran razón que se guarden mucho, ainsí en lo que han de comer como de vestir, e de todas las cosas les sean fecha salua. Ca no es marauilla que los moros vsen dello, que ya los malos cristianos lo usan, queriando mal a otro, o por alcançar su honrra e estado, luego piensan de lo matar con yervas. » Ibid. 8, p. 271.

12. «Estando así el Rey en Valencia, adolesçió de dolor de hijada muy gravemente, é un hijo de un ama suya le dixo que él había tenido aquella enfermedad, é había sanado con agua de beleño sacado por alquitara bebida tres veces de tercero en tercero día, é con esto había sanado otros tres ó quatro enfermos desta enfermedad; y el Rey quiso saber dellos si era verdad, los quales les respondieron que sí, é que convenía que todos los todos los nueve ó diez días bebida aquella agua, estuviese en la cama ; é como quiera que los físicos lo requirieron é amonestaron que no bebiese aquella agua, diciéndole como era cosa cosa muy fuerte é que aquellos que habían sanado con ella eran hombres robustos é de más fuerte complesión que él, é que por eso que en ninguna manera le debía beber, y el Rey todavía quiso probar en sí esta experiencia, é bebida el agua no dexó de se 
levantar, y echado un día en su cámara él se amontesció de tal manera, que estuvo sin pulsos mas de una hora, é por toda la cibdad fue fama que era muerto, é porque creyesen el contrario lo pusieron á una ventana de su Palacio porque todos lo viesen, é después que esta agua el Rey bebió, nunca estuvo bien sano hasta que murió, é algunos dicen que le fueron dadas yerbas, é otros dicen esto haber seydo la causa de su muerte. », Crónicas de los Reyes de Castilla, vol. 2, p. 363. 13. «O fagan matulas de beleño y seuo de carnero y de cuestas: y matulilla de empuxo son estas. " Juan de Burgos, Sevillana Medecina de Juan de Aviñón, BNM R/30652, fol. 102rº ; publié par Eric Naylor (Madison, Hispanic Seminary of Medieval Studies, 1995), REAL ACADEMIA ESPAÑOLA : Bancos de datos (CREA). Corpusde referencia del español actual. http://www. Raes.es.

14. «Capítulo. Para el dolor del vientre. E dize galieno que para/ esta enfermedad sanar virt[u]des del beleño Toma/ las fojas \& majalas \& ayuntadas/ conla farina del ordio o/ çauada. que todo es vno \& fazlo/ enplasto \& ponlo sobre/ las finchaduras \& sanara/ e toma 1 çumo \& metelo en/ las orejas que tienen dolor \&/ sanaran Otrosy toma la/ semjente \& la raíz desta dicha yerua \& cuezelo con vinagre/ e metelo enla boca fasta que faga/ espuma \& sanara el diente/ que duele Otrosy toma el çumo/ desta yerua \& echalo enlos/ ojos \& sanaran. Otrosy toma/ el çumo desta yerua \& echalo enlos/ ojos \& sanaran. Otrosi toma/ el çumo desta dicha yerua \& ayuntalo con/ farina de ordio \& faz enplasto/ \& ponlo sobre los compañones/ del omne que estoujeren jnchados/ o sobre las telas mujeres/ \& sanaran/ e faz venjr las carnes Nuevas. Fol. 12R.» Enríquez, Juan, Secretos de la medicina, Madrid, 1471, Palacio II/3063, CORDE.

15. Santa Cruz, Alonso de, Crónica de los Reyes Católicos, Juan de Mata Carriazo, Séville, Escuela de Estudios Hispano Americanos, 1951, p. 1491-1516.

16. «Y estando la corte en esta villa, por el mes de março, y el rey don Fernando en Carrioncillo, lugar apartado de Medina por una legua, deleitoso y de mucha caça, holgándose con la reine Germana su muger; donde como Su Alteza tuviese tanto deseo de tener generación, principalmente un hijo que heredase los reinos de Aragón, le hiço dar la Reina algunos potajes hechos de turmas de toro y cosas de medecina que ayudavan a hacer generación, porque le hicieron entender que se empeñaría luego. Aunque otros pensaron que les avían dado veneno, o tósigo.»Ibid., p. 281.

17. C.O.R.D.E : Corpus Diacrónico Del Español, Real Academia Española. http://corpus.rae.es/ cordenet.html.

18. Francisco López de Villalobos, Sumario de la medicina con un compendio sobre las pestíferas bubas, Madrid, BNI, p. 1169.

19. «Fen vj. De los venenos y ponçoñas/ Qualquiera ponçoña contraria y maligna o haze aquel daño con su calidad asi como arsénico y liebre marina o sin calidad ala muerte camina como es el napelo con su propriedad aquel que ponçoña a tomado o beuido el siente vn sabor y vn aborrible congoxas y arcadas y el color perdido si es fría ponçoña esta todo adormido con gran frialdad perezoso immouible/ Prosigue las señales y pone la cura/ Gran sed gran congoxa gran fiebre y ardor/ y rabia terna si ponçoña es caliente/ do no siente daño de frío o calor/ mas tiene desmayo flaqueza y sudor/ es porque con su propiedad le atormente/ con agua caliente y azeyte qualquiera/ hazed reuersa al que tiene el veneno/ si el vientre de baxo del daño se altera/ echadle vna ayuda en qualquiera manera/ después metridato beuido es muy bueno/ Prosigue la cura/ Y si la ponçoña se siente quemar/ gran golpe de leche le den a beuer/ y agua esfriada le deuen de dar/ con canfora el vientre aplastar/trosciscos de rosas le hagan comer/ si es frio el veneno tras lo vniuersal/ le den a comer muchos ajos y vino/ si con propiedad a causado este $\mathrm{mal} /$ triaca para esto es muy especial/ y con su cordiales le esfuerça continuo/ De la ponçoña $\mathrm{del} /$ perro rabioso $\mathrm{u}$ otro qualquier animal de aquella manera/ Si algún animal enponçoña y estraga/ o perro rabioso asi curad/ dexar harta sangre salir de llaga/ si fuere pequeña mas grande se haga/ y todas sangrías y purga dexad/ metridato y triaca en brebajo le dad/ la llaga emplastadrad desque bien desangrada/ con ajo y cebollas y desque en verdad/ es dentro el veneno dalla le sacad/ con purgas y asi como a la antepassada/ Prosigue./ Y en días quarenta no 
hagays soldacion/ de la dicha llaga y ligad fuertemente/ en medio daquesta y del coraçon/ y en vn año todo le dad su racion/ de algún bezariaco o triaca potente/ y de vulgar/ con armas como estas quisiere matar/ no quiero hazergelo aquí manifiesto. »

20. Enrique de Villena, Arte Cisoria, P. Catédra (éd.), Turner, Madrid, 1994.

21. "guarnidas sus manos de sortijas que tengan piedras o encastaduras valientes contra ponçoña e aire infecto, ansí como robí e diamante e girgonça e esmeralda e coral e unicornio e serpentina e bezuhar e pirofilis, la que se faze del coraçón del omne muerto con veneno frio e cocho, siquiere enduresçido o lapidificado en fuego reverberante. Esta traída Alixandre sobre todas consigo, segúnt aristóteles en su Lapidario cuenta. ». Ibid, p. 143-144.

22. «E tal vaina tenga cobertura como escrivanía con sus cordones fermosamente labradas. E puéndenlos fazer de cuero; e si fuese çervuno, sería mejor, porque es contra veneno, e por eso fazen con él las ataduras en las mordeduras venenosas. » (p.153).

23. Mayerne Turquets, Loys de, Histoire générale d'Espagne : comprise en XXX libres, Paris, Chez Abel l'Angelier, 1608.

24. Bofarull, Antonio, Historia crítica (civil y eclesiástica) de Cataluña, Barcelone, Juan aleu y Fugarull (éd.), 1876-1878.

25. Pelta, Roberto, El veneno en la historia, Madrid, Espasa-Calpe, 1997.

26. "Se cuenta a este respecto que en una ocasión su hermanastro, el futuro rey Fernando el Católico, tuvo que 'hacer la salva' al negarse Carlos de Viana a comer; el verbo 'salvar' hace alusión a la costumbre de probar la comida y la bebida por parte de una persona encargada de dar servicio a los reyes y grandes señores, a fin de descartar la existencia de veneno en las mismas. ", Ibid., p. 204.

27. À la suite d'une consultation de C.O.R.D.E.

28. García de Salazar Lope, Historia de las biendanzas e fortunas, Ana María Sanchez, Corde (Madrid), 2000.

29. «Contado ha la historia cómo morió el rey don Alonso en el Real de Gibraltar e cómo los castellanos salieron con el cuerpo e cómo luego començaron bullicios. En el primer año de su reinamiento fue presa doña Leonor de Guzmán, mançeba que fuera del rey don Alonso e madre de sus fijos don Enrique e don Fadrique e don Tello e don Sancho, en Sevilla por la reina doña María, por consejo de don Juan Alonso de Alborquerque, mayordomo del Rey, que era pariente de la Reina. Fue levada al Castillo de Talavera e después muerta allí con yerbas. E luego que ella fue presa, salieron de la corte sus fijos e sus parientes, los de Guzmán, por donde se siguió mucho mal en Castilla en el tiempo d'este rey don Pero, segund adelante se dirá. » (1370).

30. «Título de cómmo el rey don Pero fizo matar a la Reina de Aragón e a doña Juana, señor de Vizcaya e de Lara. En el nobeno año del reinado d'este rey don Pero, que fue en el eaño del Señor de mil CCCLXIX años, fizo matar en Castroxeriz a la Reina de Aragón, que estaba allí presa por despecho, qu'el infante, su fijo le fazía guerra, e fizo matar con yervas a doña Juana de Lara, muger del conde don Tello, Señora de Vizcaya, e doña Isabel, su hermana, muger del infante don Juan, en el Castillo de Almodóvar del Río, que estavan presas. E en Blanca de Borbón, su muger, que estava en el castillo de Medina Sidonia. » Fol. $317^{\mathrm{v}}$.

31. Crónica del rey Juan I, p. 135-136.

32. «Muertes de la reina de Portugal y Castilla, hermanas del rey [de Aragón] ; las sospechas que causaron y con qué indicios. Había fallecido a 18 del mes de febrero deste año la reina doña Leonor de Portugal estando en la ciudad de Toledo en el monasterio de Santo Domingo el Real ; y llegó al rey de Castilla la nueva de su muerte estando en El Espinar. Y pocos días después que el rey de Navarra pasó los puertos murió la reina de Castilla en Villacastín, aldea de Segovia. Y tuvieron por cierto las gentes que les fueron dadas yerbas; y dellos hubo muchas señales, y fue inculpado el condestable don Álvaro de Luna que con su sabiduría y consejo se aparejó el veneno con que murieron las reinas y que fue manifiestamente entendido por la repentina muerte de la reina de Portugal y por la celeridad del veneno con que murió, y que todos los indicios y señales 
dél se vieron en su cuerpo siendo defunta, y que con el mismo fue muerta la reina de Castilla su hermana. Y dio mucha ocasión desta fama que en lo de la honestidad de la vida destas princesas se pudiera haber hablado y juzgado mejor y en la reciente confederación que hubo de la reina de Castilla con el príncipe su hijo para sacar al rey su marido del poder de su condestable. » (Libro XV, p. 333-334), [1445].

33. «Sentimientos del rey [de Aragón] por las muertes de las reinas sus hermanas. Pero cuando el rey supo lo que se publicó de la muerte de las reinas sus hermanas recibió gran pena dello y envió a mandar a sus embajadores que si fuese así que la fama que por todas partes se divulgaba fuese verdadera, que las muertes de las reinas de Castilla y Portugal se habían seguido con industria y maldad, no tratasen de las cosas contenidas en las instrucciones que traían. Esto fue estando el rey en Nápoles a 27 del mes de mayo, ocho días después de haberse puesto ya el estado de todas las cosas a juicio y trance de batalla, antes que llegasen sus embajadores. » (Libro XV, p. 334).

34. «Titulo de la prisión e muerte del adelantado Pero Manrique. En este año sopo el rey don Juan cómo el adelantado Pero Manrique se trataba con el don Alonso de Gijón e con otros algunos por poner bullicio en el reino. E por esto e porqu'él era ome bulliçioso e gastador demasiadamente e ponía mal recaudo en su fazienda e no avía fijos, fizolo prender el Rey. E estoviendo preso en el castillo de Plazençia, fue muerto a cavo de un año; e díxose que de yerbas e que fueran sus hermanos Gómez Manrique e Diego Gómez Manrique en el consejo de su muerte e que por ello les dio el Rey todos sus heredamientos. » (environ 1380).

35. «Sobre estas cosas fechas en Castilla, juntáronse los reyes don Alonso de Aragón e don Juan de Navarra e infante don Enrique e el Conde e otros cavalleros algunos de Castilla que eran de su favor e entraron por Castillas; e salieron el dicho Condestable e los otros caballeros del reino a ellos e juntáronse en Monteagudo para pelear; e estando allí, ovieron sus tratos de paz. E tornados cada unos a sus tierras, el rey don Juan toviéndose por injuriado porque los Reyes de Aragón e de Navarra le avían entrado en sus reinos, € juntó sus gentes e fijosdalgo, que fueron muchos además, para entrar en Aragón e puso su real en Veramaçán. E allí fue preso el Duque de Arjona, veniendo de Galiçia con mil de cavallo e mucha gente de pie, deziendo que avía seido de fabla de la entrada de los eyes de Aragón e de Navarra, e fue puesto en el castillo de Peñafiel e allí morió de yerbas. Entró el dicho rey don Juan por Aragón e, fecho algunos daños en él e tornado en Castilla, repartió todos los eredamientos que los Infantes e Duque tenían en Castilla en los caballeros del reino. E seguíase la guerra. »

36. «Título de cómo fue preso el rey don Juan en Medina del Canpo e fuyó el Condestable a Escalona. En esta sazón fue preso el adelantado Pero Manrique e salióse de Fuente Dueña; e juntándose con los caballeros, fizieron salir al Condestable de corte e estuvo en Escalona fasta que ellos mesmos gelo fezieron traer al Rey. E venido, fueron dadas yervas al dicho Adelantado comiendo con el Arçobispo de Toledo, díxose que por mandado del Maestre, su hermano, e morió d'ellas por luenga dolencia. »

\section{RÉSUMÉS}

Durant les derniers siècles du Moyen Âge, le poison semble avoir joué un rôle important dans la vie politique castillane, et plusieurs auteurs de chroniques ou de traités médicaux ont prêté attention à la réalité de ce phénomène. Notre article se centrera sur l'usage du poison chez les Trastamare et ses conséquences politiques. Nous souhaiterions montrer l'impact du poison dans 
l'imaginaire des contemporains et la réalité de cette pratique dans la dynastie Trastamare. Le présent article s'attachera enfin à démontrer que les références au poison sont avant tout des armes propagandistiques au service du pouvoir.

During the last centuries of the Middle Ages, poison seems to have played an important role in Castilian political life, and many authors of chronicles and medical treatises pay attention to the reality of this phenomenon. The article focuses on the use of poison by the Trastamaras, and on its political consequences. We attempt to show the impact of poison on the imagination of contemporaries and on the reality of this practice by members of the Trastamara dynasty, and to demonstrate that references to poison are essentially propagandandistic arms in the service of power.

\section{AUTEUR}

\section{FLORA RAMIRES}

Université Paris-III Sorbonne nouvelle. 